\title{
CFD Investigation of Rotational Sloshing Waves in a Top-Submerged-Lance Metal Bath
}

\author{
D. OBISO, M. REUTER, and A. RICHTER
}

Computational fluid dynamics (CFD) is applied to investigate rotational sloshing waves in a top-submerged-lance (TSL) cylindrical metal bath. The study is an extension of a recent work of the authors, where the top injection of Ar into a metallic bath was examined in a quasi-2D flat setup, allowing the numerical model to be extensively validated against experimental data based on x-ray radiography. The new analysis of top gas injection in a cylindrical vessel reveals the appearance of rotational sloshing in the bath, which is maintained by a condition of synchronism between the gas bubbles and the free surface of the bath. A numerical quantification is achieved with specific post-processing of the simulation results, showing the effect of control parameters such as the lance immersion depth and the gas flow rate. This fundamental research study demonstrates the capability of CFD modeling to predict bath dynamics known from literature and practice, the understanding of which is essential for the design of TSL furnaces.

https://doi.org/10.1007/s11663-021-02182-x

(C) The Author(s) 2021

\section{INTRODUCTION}

THE presence of rotational sloshing waves in cylindrical liquid baths has been observed in various fields of application. ${ }^{[1-6]}$ When the natural sloshing mode of the bath is in resonance with the mode of an external perturbation, the free surface of the liquid may form a slope and rotate around the axis of the vessel. Early investigations were carried out in space technology, in which the liquid fuel of a liquid-propelled rocket can enter in resonance with the tank vibrations, leading to rotary sloshing. In theoretical and experimental work, Hutton proposed an analytical expression, which sets an upper limit to the net angular momentum of the liquid and can be used to estimate the induced swirling velocity. ${ }^{[7]}$ This method was later used to study gas-stirred tanks in metallurgical processes, where the perturbation of the system consists in the submersed injection of gas into a heavy liquid. Schwarz theorized the mechanism of rotary sloshing for vessels with bottom gas injection. ${ }^{[8,9]}$ The rotary waves appear when the motion of the bubble plume is in resonance with the

D. OBISO is with the CIC Virtuhcon, TU Bergakademie Freiberg, Fuchsmühlenweg 9, 09599 Freiberg, Germany. Contact e-mail: daniele.obiso@iec.tu-freiberg.de M. REUTER is with the SMS group GmbH, Eduard-Schloemann-Str. 4, 40237 Düsseldorf, Germany. A. RICHTER is with the Chair of Energy Process Engineering, TU Bergakademie Freiberg, Fuchsmühlenweg 9, 09599 Freiberg, Germany.

Manuscript submitted January 12, 2021; accepted April 9, 2021.

Article published online May 17, 2021. motion of the free surface. Under those circumstances, the bath starts first to oscillate in a two-dimensional motion. However, in cases of axial symmetry, as with cylinders, there is no preferred two-dimensional wave and the energy can be transferred from one to the other wave, resulting in a rotative motion.

Rotary sloshing was also observed in top-submerged-lance (TSL) furnaces, where the process gas is injected downwards into the liquid with a top lance. The presence of these wave motions generates high forces and torques on the structure of the furnace itself; as a result, the foundations of the building and the furnace support system need to be designed carefully. ${ }^{[10]}$ However, little fundamental research is found in the literature. Liow et al. experimentally investigated sloshing phenomena for Peirce-Smith bath types. ${ }^{[11,12]}$ Using a water-air model, he observed the presence of rotating waves on the free surface, whose amplitude increased with the gas flow rate and lance immersion depth. Unlike a monotonic increase with the gas flow rate predicted by Schwarz, he observed the achievement of a plateau and later the disruption of the waves at the highest rates. It must be mentioned that Schwarz's model, based on a linear approximation of the momentum equations, is valid only under the assumption of relatively small wave amplitudes, surely not the case in Liow's tests. The wave amplitude also decreased at the lowest lance position. The lower and upper limits of the gas flow rate and lance immersion set the boundaries for the resonance condition and therefore the appearance of the sloshing. In another work, ${ }^{[13]}$ Liow et al. also carried out an experimental investigation to study the damping 
effects of sloshing waves generated by top gas injection. They added a thin layer of paraffin oil to the water-air model to understand whether the presence of a viscous layer in the bath could prevent the bath slopping. The outcomes showed that rotating waves were significantly damped in the presence of the oil layer, since the bath oscillation did not have a primary sloshing frequency, but rather a broad spectrum of frequencies. Wang et al. carried out a Computational Fluid Dynamics (CFD) simulation of top-submerged gas injection for a slag stabilization process. ${ }^{[14]}$ Among other things, they analyzed the sloshing waves at the free surface, monitoring the slag volume fraction at points close to the interface. It was found that the amplitude of the wave increases with the lance submersion depth, whereas its frequency stays constant. However, the vessel they examined has a vessel-to-lance-diameter ratio $>70$, which is relatively high for their conclusions to be extended to TSL smelting furnace types, which are the background reference of this work.

In a recent publication, the authors presented a numerical and experimental study of TSL gas injection in a liquid metal bath. ${ }^{[15]}$ The work offers a detailed insight into the multiphase flow generated by the top injection of Ar gas into a liquid metal bath and proves the reliability of the Volume of Fluid (VOF) modeling approach for complex fluid systems. The ternary alloy GaInSn was used as a fluid model, whose eutectic point at room temperature facilitates the measurement procedure. $^{[16]}$ The combination of $\mathrm{x}$-ray radiography and CFD simulation directly reveals flow features such as void fraction distributions, bubble size and detachment frequency, and break-up mechanisms, which are otherwise difficult to detect for opaque fluids. Nevertheless, the use of a flat quasi-2D vessel still limits the analysis, since inherently $3 \mathrm{D}$ flow structures, such as the slope sloshing, cannot be reproduced. In fact, the presence of the side walls restricts the bubble dynamics and leads to steady recirculation vortexes in some of the configurations, blocking the flow with asymmetric patterns.

The present work is intended to be an extension of that study, ${ }^{[15]}$ and investigates top gas injection in $3 \mathrm{D}$ cylindrical vessels by means of CFD modeling. This allows eventual sloshing phenomena to be tracked and examined. The same Ar-GaInSn fluid system is adopted, whose physical properties are reported in Table I.

Table I. Physical Properties of the Eutectic Alloy GaInSn at Ambient Temperature

\begin{tabular}{lll}
\hline Property & \multicolumn{1}{c}{ Value } & Unit \\
\hline$\rho$ & 6360 & $\mathrm{~kg} / \mathrm{m}^{3}$ \\
$\mu$ & $2.16 \times 10^{-3}$ & $\mathrm{~kg} / \mathrm{ms}$ \\
$\sigma$ & 0.533 & $\mathrm{~N} / \mathrm{m}$ \\
\hline
\end{tabular}

\section{NUMERICAL SETUP}

Figure 1 shows the computational domain used in this study, a cylinder with a vessel-to-lance-diameter ratio slightly $>10$, as in common TSL smelters. ${ }^{[17]}$ The metal bath has a height $H$ and diameter $D$, which are both equal to $60 \mathrm{~mm}$. The lance specifics are the same as in Reference 15, namely $5.5 \mathrm{~mm}$ for the outer diameter and $5 \mathrm{~mm}$ for the inner diameter. Six different configurations were studied, varying the immersion depth of the lance and the gas volume flow. The three positions of the lance, labeled top, middle, and bottom, refer to immersions at $1 / 4 \mathrm{H}, 1 / 2 \mathrm{H}$, and $3 / 4 \mathrm{H}$. The first $\mathrm{Ar}$ volume flow of $Q_{1}=0.110 \mathrm{l} / \mathrm{s}^{[15]}$ was increased to $Q_{2}=0.165 \mathrm{l} / \mathrm{s}$ and $Q_{3}=0.220 \mathrm{l} / \mathrm{s}$ for the middle lance position configuration. These cases will be labeled $Q_{1}$, $Q_{2}$, and $Q_{3}$. The numerical model is based on the VOF method, and the setup is reported in Table II. For the sake of simplicity, the mathematical formulation is not reported here, and the reader is referred to Reference 15. Here, a grid convergence study also showed that a space

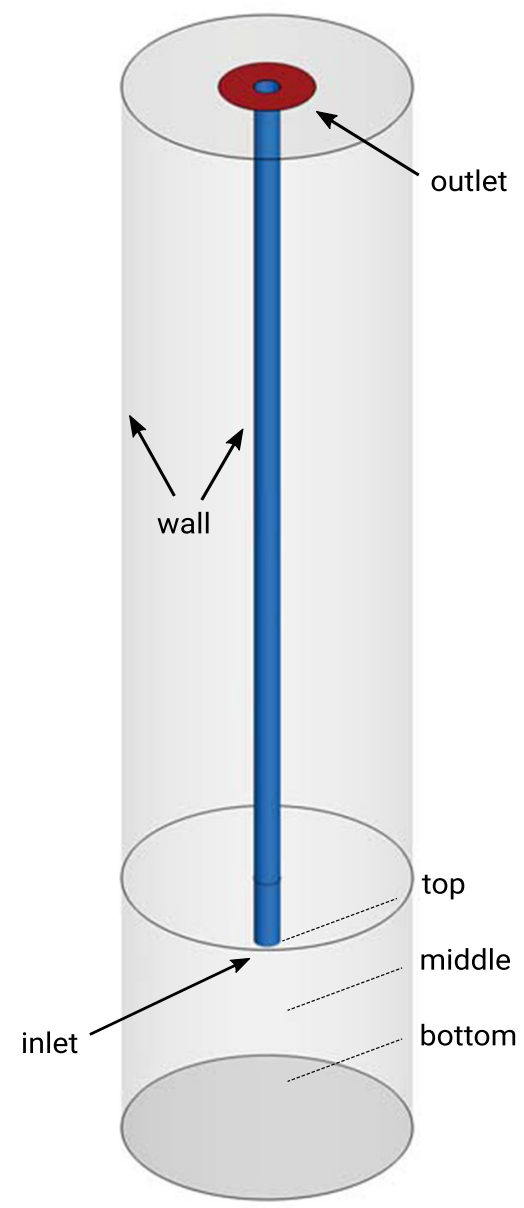

Fig. 1-Computational domain and boundary conditions used for the simulations. The captions top, middle, and bottom refer to the three analyzed lance positions, at $1 / 4 H, 1 / 2 H$, and $3 / 4 H$, where $H$ is the height of the bath: $0.06 \mathrm{~m}$. 
resolution in the order of $10^{-4} \mathrm{~m}$ provided mesh-independent results. Hence, the same mesh structure and resolution are adopted in the present work.

The commercial software ANSA Beta CAE (v.18) was used to mesh the domain with a structured hexagonal approach. The hexa-block structure of the grid is shown in Figure 2, in which a $z$-normal slice reveals the inner distribution of the cells. As can be seen, except for the obvious refinement of the injection area, the spatial discretization is kept constant in the domain to ensure the same degree of resolution for the geometric reconstruction of the gas-liquid interface. The grids have a size of approximately $2 \mathrm{M}$ cells, and the simulations were run to complete the calculation of 24 seconds of real time. In order to exclude the initial transient development of the flow, the first 4 seconds of the simulation are removed from the statistical analysis of the results, which therefore covers 20 seconds of real injection time. The simulations are carried out using the

Table II. Numerical Setup

\begin{tabular}{ll}
\hline Solver & 3D simulation \\
& VOF-explicit \\
& SIMPLE p-u coupling \\
& Continuum Surface Force \\
& model for $\sigma$ \\
& $k \epsilon$-Realizable \\
Turbulence & Second-order upwind: \\
Numerics & momentum \\
& PRESTO!: pressure \\
& First-order upwind: turbulence \\
& for $\alpha$-Reconstruct algorithm \\
& Minimum grid size: $1.5 \cdot 10^{-4}$ \\
& m \\
Dime step: $1 \cdot 10^{-5} \mathrm{~s}($ Courant \\
\end{tabular}

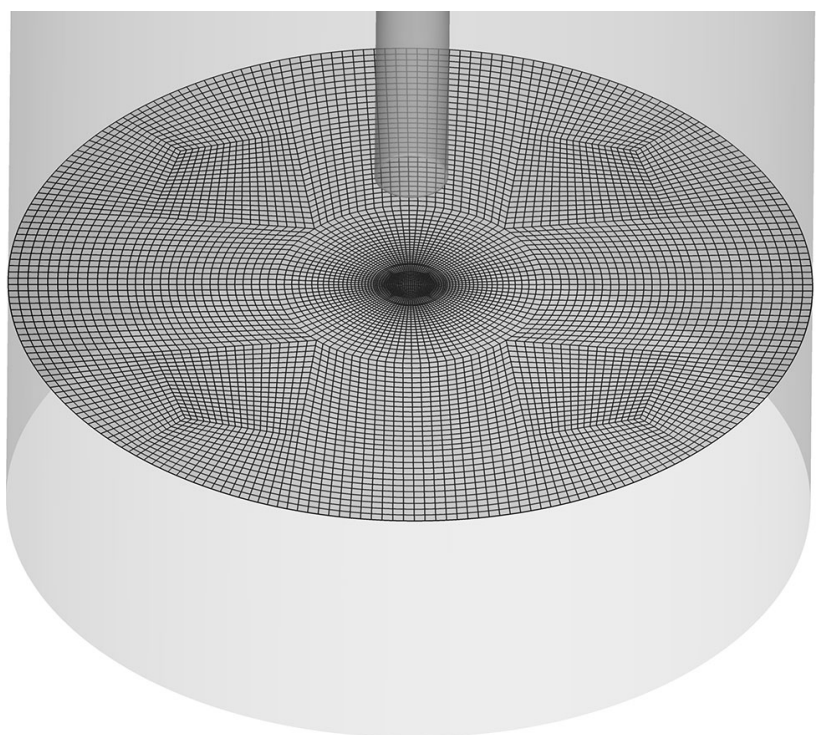

Fig. 2-Inner structure of the computational grid, shown on a $z$-normal slice. A hexa-block approach is applied to generate the structured mesh. commercial software ANSYS Fluent (v.19.2) on 288 CPUs, allocated at the HPC Cluster Center for Information Services and High Performance Computing (ZIH) at TU Dresden, and the calculation of the elapsed time is completed with a number of computing days of $\mathrm{O}(10)$. To monitor the sloshing behavior during the simulation, the Center of Mass (CoM) of the liquid phase is computed and tracked over time with a user defined function (UDF). Commonly used in computational slosh dynamics for automotive and aerospace fuel tanks, ${ }^{[18]}$ this procedure allows the liquid bath motion to be monitored. Indeed, when the bubble rises up with a radial displacement from the lance, the free surface shows an incline, which is higher on the side of the bubble. It is precisely the synchronism between the bubble and free surface motions that originates and sustains the sloshing. ${ }^{[8]}$ Figure 3 displays a snapshot of the gas-liquid interface for the middle configuration; the interface is visualized with a volume fraction iso-surface equal to 0.5 . The relation between the slope and bubble position is clearly visible, confirming what was described by Schwarz. In this bath configuration, the CoM of the liquid phase moves from the centerline towards the angular position with the taller free surface. As a consequence, the sloshing dynamics can be tracked by monitoring the CoM position over time.

\section{RESULTS AND DISCUSSION}

\section{A. Rotational Sloshing}

Figure 4 shows an example of the time development for the CoM angular position $\Theta$ and the angular velocity $\omega$ for the top configuration. The angular position clearly shows the rotation of the liquid bath,

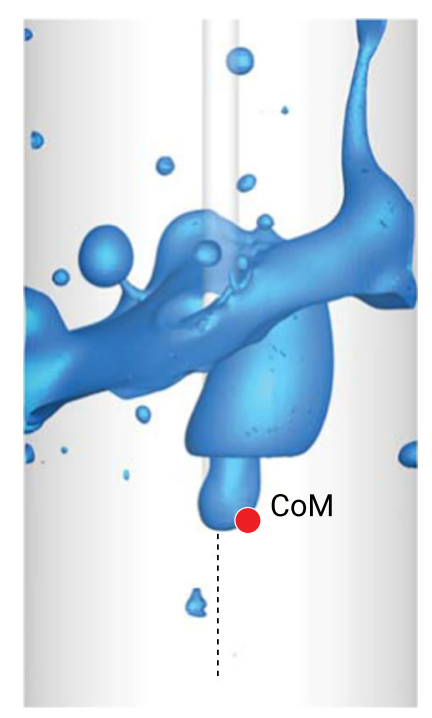

Fig. 3-A snapshot of the gas-liquid interface for the middle configuration. The slope of the free surface is seen to be synchronous with the bubble direction, as theorized by Schwarz. ${ }^{[8]}$ The calculation of the liquid's Center of Mass (CoM) therefore allows the sloshing to be tracked, since it moves away from the centerline during the oscillations. 
with a relatively constant angular velocity. It is evident that the rotational sloshing is already active after the first 4 seconds of injection and then maintained in a steady condition by the resonance between the bath and bubble plume modes. The same information can also be qualitatively visualized, plotting the location of the $\mathrm{CoM}$ in the $X Y$ plane over time. Figures 5(a) through (c) respectively illustrate the results for the top, middle, and bottom configurations with the gas flow $Q_{1}$. The rotation of the liquid around the axis is again visible in all the cases. It is interesting to note that, except for short time ranges, the CoM proceeds with almost circular motions. Furthermore, the displacement from the axis increases with the lance submersion, which can be translated into an increase in the sloshing wave amplitude. This can also be observed in the temporal evolution of the gas-liquid interface, which is available as a movie sequence and has been attached to this article as Electronic Supplementary Material. In Figures 6(a) through (c), the CoM motion is reported for the cases $Q_{1}, Q_{2}$, and $Q_{3}$. Although rotations of the CoM can be discerned, the path becomes more chaotic when the gas volume flow is increased, and it is arduous to extract qualitative information on the trend.

To quantify the sloshing effect induced by the gas injection, the time-averaged swirl component of the velocity is computed in each cell of the domain as

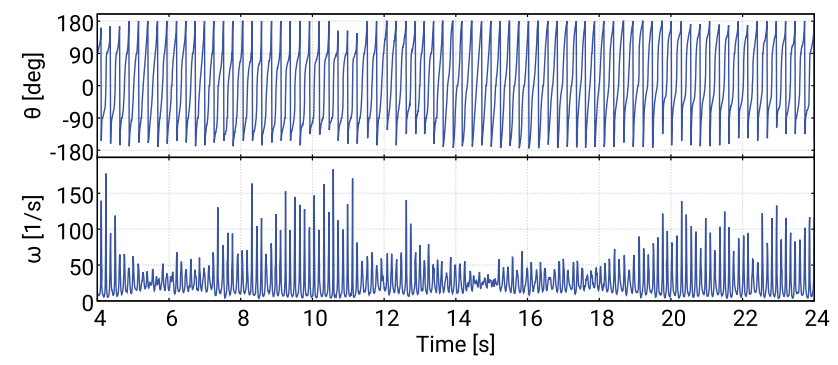

Fig. 4-Angular position and velocity of the CoM over time for the top configuration, indicating the presence of rotational sloshing.

$$
\left\langle v_{\text {swirl }}\right\rangle=\frac{1}{T} \int_{0}^{T} v_{\text {swirl }} d t
$$

The spatial distribution of $\left\langle v_{\text {swirl }}\right\rangle$ is then filtered, blanking the regions where the time-averaged liquid fraction is $<0.5$. The selected domain represents the region occupied by the liquid phase averaged over time. Figures 7(a) through (c) and 8(a) through (c) display contour distributions of the time-averaged swirl velocity in the bath for all the configurations: top, middle, bottom, $Q_{1}, Q_{2}$, and $Q_{3}$. The contour maps are reported over bath slices, showing the radial and axial distribution of $\left\langle v_{\text {swirl }}\right\rangle$. To help picture the domain, the time-averaged gas-liquid interface is additionally shown in green. Qualitatively, it can be seen that lowering the lance into the bath enhances the induced swirl and moreover pushes the circular motion to the bottom of the vessel. As a consequence, the stir in the liquid phase is favored. The same can be observed when the gas volume flow is increased from $Q_{1}$ to $Q_{2}$, whereas the distribution for the case $Q_{3}$ does not exhibit any continuity in its behavior. The time-averaged distributions of $\left\langle v_{\text {swirl }}\right\rangle$ show symmetric patterns in the liquid bath, whereas local asymmetries are detected in the bubble plume area of some configurations. The reason is the rather chaotic nature of the flow in this region, which a 20 -s time observation window might not adequately average. The simulation of a longer elapsed time is not feasible with the currently available computing resources; yet, according to the authors, the outcomes of the study are not significantly affected by this.

Applying a volume average to the 3D distribution of $\left\langle v_{\text {swirl }}\right\rangle$ produces a mean value (in time and volume) of the induced swirl velocity in the liquid phase, which can be used to quantitatively compare the configurations. The results of the analysis are summarized in Figure 9. Moving from 25 to 75 pct bath submersion, the swirl velocities are more than quadrupled from $0.0039 \mathrm{~m} / \mathrm{s}$ to $0.0175 \mathrm{~m} / \mathrm{s}$ with a monotonic increase, together with the sloshing, as previously disclosed. This is in agreement

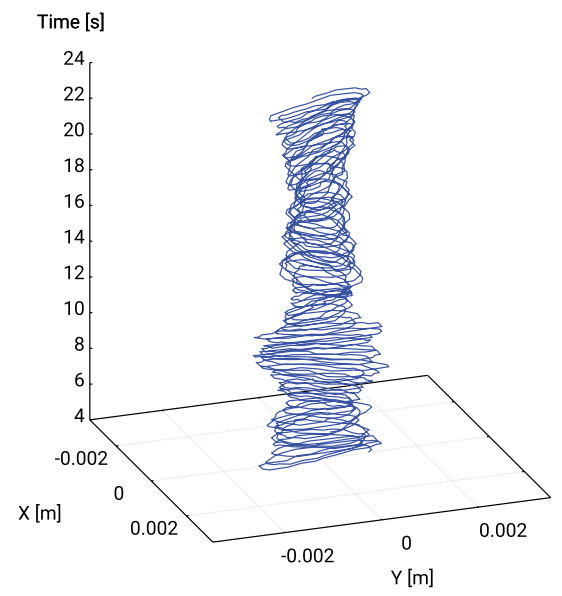

(a)

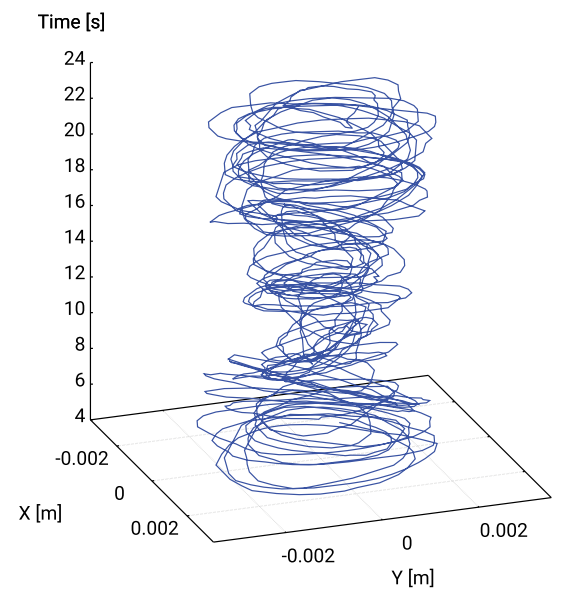

(b)

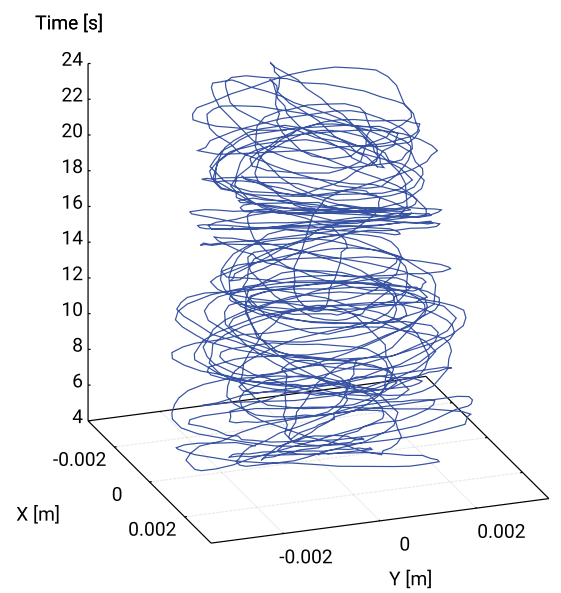

(c)

Fig. 5-Position of the CoM in the plane $X Y$ over time for the (a) top, (b) middle, and (c) bottom configurations. 


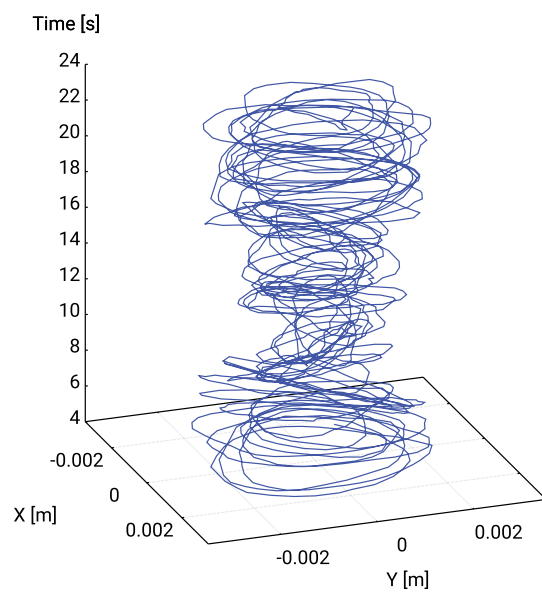

(a)

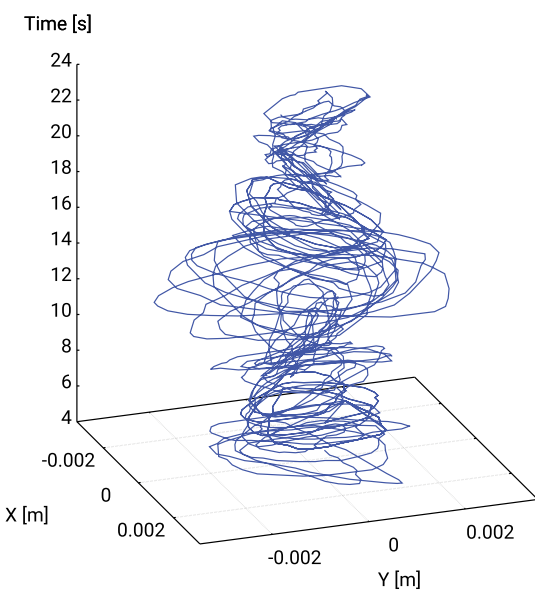

(b)

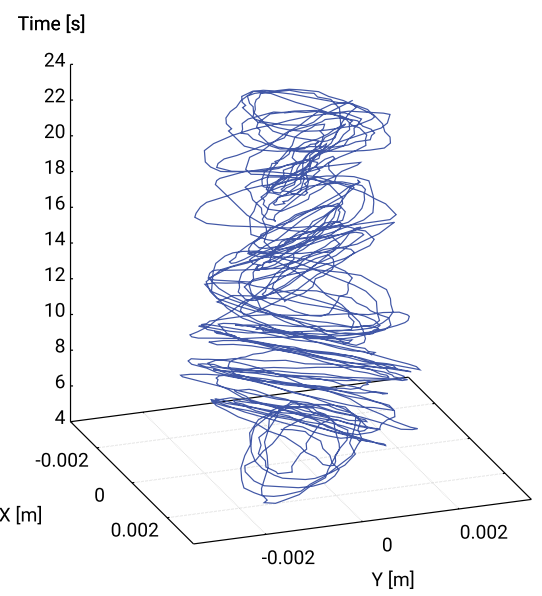

(c)

Fig. 6-Position of the CoM in the plane $X Y$ over time for the (a) $Q_{1},(b) Q_{2}$, and (c) $Q_{3}$ configurations.

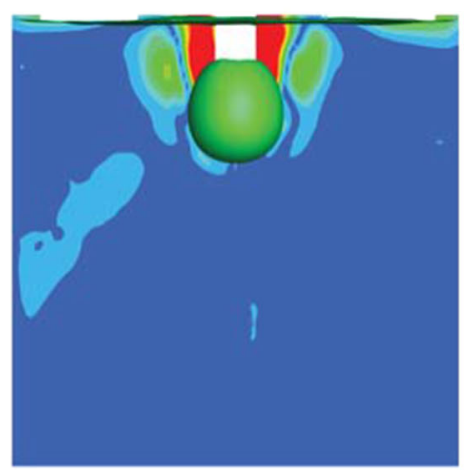

(a)

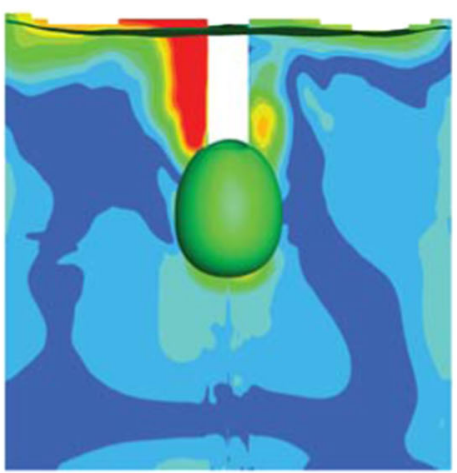

(b)

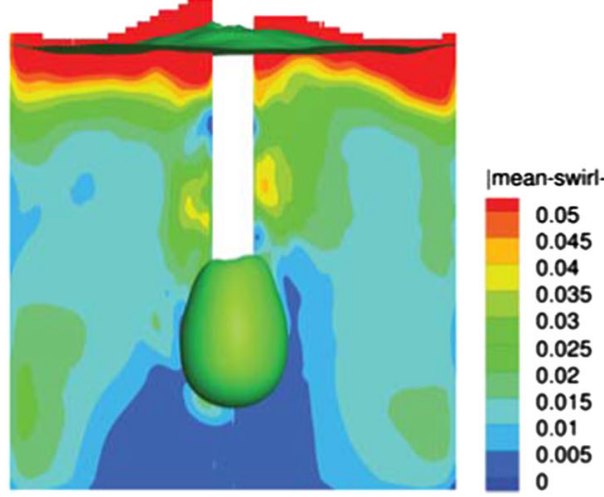

(c)

Fig. 7-Contour distribution of the time-averaged swirl velocity in the liquid phase for the (a) top, (b) middle, and (c) bottom configurations. The areas occupied on average by the gas phase are blanked so that the focus is only on the liquid phase, and the visualization of the time-averaged interface (green surface) helps to locate the boundaries (Color figure online).

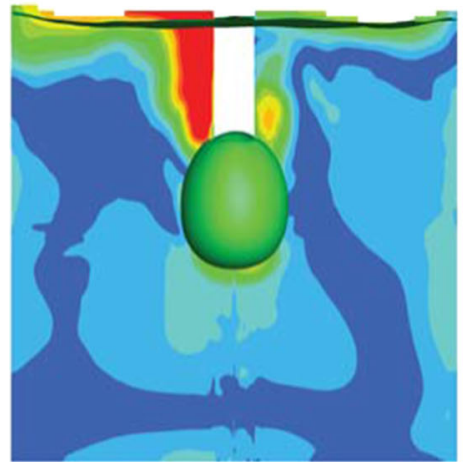

(a)

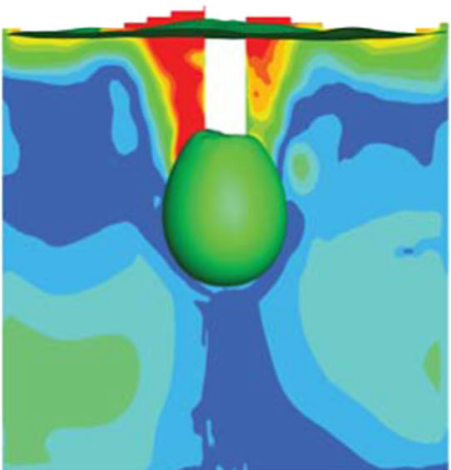

(b)

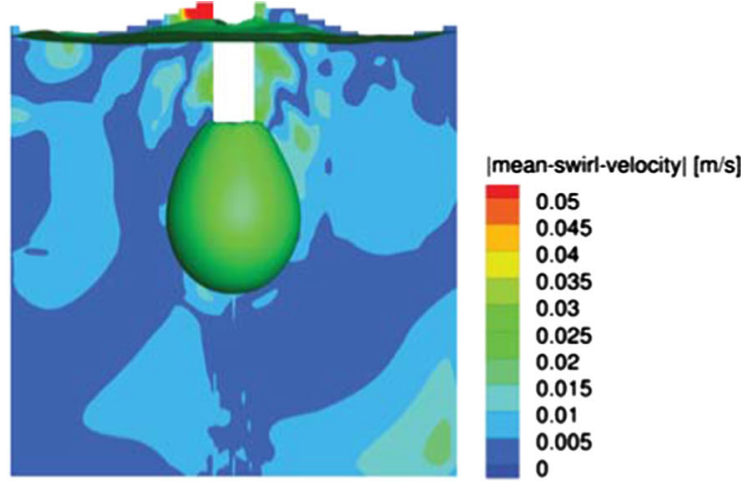

(c)

Fig. 8 - Contour distribution of the time-averaged swirl velocity in the liquid phase for the $(a) Q_{1},(b) Q_{2}$, and (c) $Q_{3}$ configurations. The areas occupied on average by the gas phase are blanked so that the focus is only on the liquid phase, and the visualization of the time-averaged interface (green surface) helps to locate the boundaries (Color figure online).

with the observations made by Liow et al. in their air-water experiments. ${ }^{[12]}$ Monitoring the wave amplitude, they found a similar behavior, interrupted at the highest tested submersion depth. In this work, 75 pct bath submersion is the maximum analyzed and the eventual peak is not reached. Nevertheless, the author 


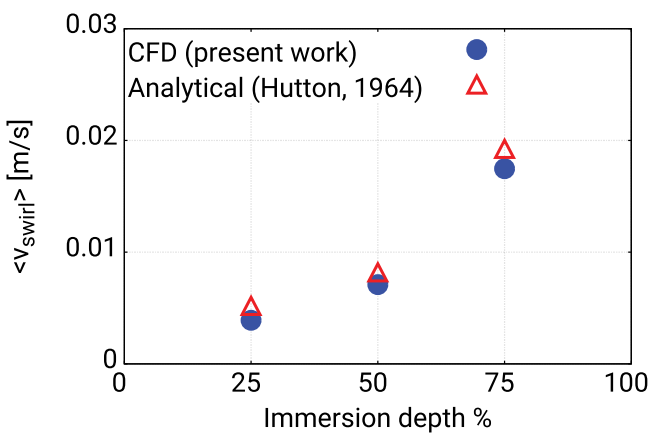

(a)

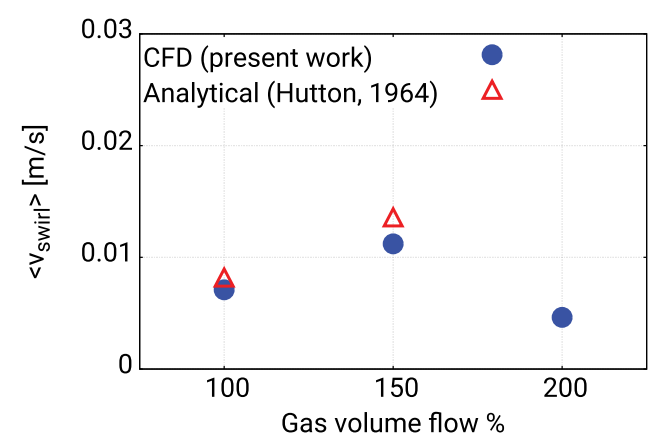

(b)

Fig. 9-Time-averaged swirl velocity induced in the liquid bath at different lance immersion depths $(a)$ and gas flow rates $(b)$. The CFD data are compared with the analytical expression proposed by Hutton ${ }^{[7]}$

believes that further submersion will not produce additional swirl in the bath. When the gas flow rate is increased, an initial increase of about 66 pct in induced swirl is observed from $Q_{1}$ to $Q_{2}$, followed by an interruption at $Q_{3}$. In this case, the results again agree with the work of Liow et al., who observed the rotational wave disappearing at high gas flow rates.

It should be noted that Liow's investigations were based on the visual observation of the waves' amplitude at the free surface, without information on bath swirl velocities. In this work, the use of CFD modeling allows the rotational sloshing to be directly calculated, providing more detailed data, as the link between wave propagation and swirl velocity. The wave disruption at high gas volume flows was not truly explained by Liow. He suggests that, at these flow rates, the turbulent kinetic energy might interact with the gas-liquid interactions, making the theory of wave amplitude variation hard to predict.

In Figure 10, the time tracking of the liquid CoM shows that rotational sloshing is actually present in configuration $Q_{3}$. However, the time evolutions of both $\Theta$ and $\omega$ show that the sign of the rotation changes at specific times, resulting in a null mean value of the swirl velocity. In the 20 seconds tracked, three changes of rotational direction are observed. Although the bath is strongly turbulent at this flow rate, the change in waves' rotation can be observed from the attached videos. Compared with the other configurations, it can be seen that the detachment and return of big droplet structures strongly interfere with the interface dynamics. This hence sets an upper boundary for the resonance condition between the bubbles and bath.

\section{B. Induced Swirl Velocity}

In Figure 9, the calculated values of $\left\langle v_{\text {swirl }}\right\rangle$ are also compared with those obtained with an analytical expression proposed by Hutton. ${ }^{[7]} \mathrm{He}$ found a simple expression to set an upper limit to the angular momentum of the fluid, here adjusted for the TSL bath, ${ }^{[14]}$ defined as follows:

$$
\frac{H_{l}}{H_{0}}<0.8\left(\frac{h_{\max }}{R}\right)^{2}
$$

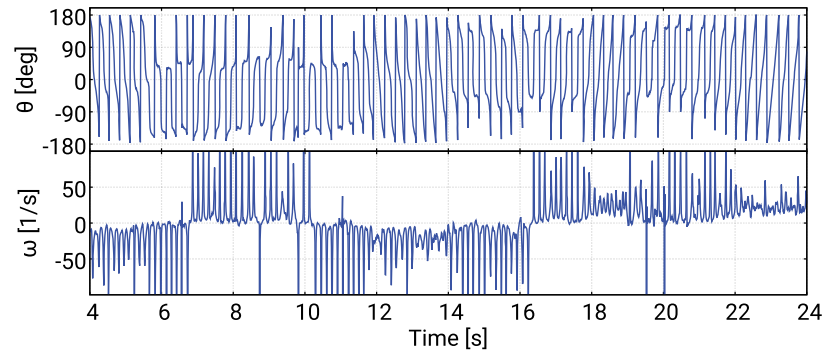

Fig. 10-Angular position and velocity of the CoM over time for the configuration $Q_{3}$. It can be observed that rotation is temporarily present in the bath. However, because of the strong multiphase interactions, the direction is inverted over time, resulting in a time-averaged drop in the swirl velocity.

where $H_{l}$ is the angular momentum of the fluid relative to the $z$-axis, and $H_{0}$ is the corresponding angular momentum the fluid would have if it rotated at $\omega$ as a rigid body. $h_{\max }$ and $R$ are the maximum height of the wave and the radius of the vessel, respectively. Expressing $H_{l}$ and $H_{0}$ as

$$
\begin{gathered}
H_{0}=\frac{\pi}{2} \rho_{\text {liq }} R^{4} \omega d \\
H_{l}=R m_{\text {liq }} v_{\text {swirl }}
\end{gathered}
$$

it is possible to estimate a maximum value of the swirl velocity of the bath $v_{\text {swirl }}$, by considering Eq. [2] as an equality. Here, $\rho_{\text {liq }}$ and $m_{\text {liq }}$ are the density and the mass of the bath, and $d$ is the submersion depth. $h_{\max }$ was extracted from visual observation of the bath's evolution. The results reported in Figure 9 show good agreement with the calculated data, also confirming the validity of the present work. The analytical expression was not applied at $Q_{3}$ since it was not possible to detect the wave propagation in this case. As Eq. [3] shows, the torque acting on the structure of the furnace is proportional to the slag density. It is evident that the design of the support system needs to be process-specific. Suffice it to mention the difference between processing a lead rich slag and a lower density one. 


\section{Liquid Splashing and Gas-Liquid Interface}

Two additional aspects of the multiphase flow were analyzed, namely the liquid splashing and the overall gas-liquid interface area. The liquid splashing on the bath surface is quantified with the evaluation of the Splashing Domain $\left(S D,\left[\mathrm{~m}^{3}\right]\right)$, as defined in the Reference 19. In brief, it represents the time-averaged volume above the free surface, which is occupied by the liquid phase. In the VOF model, the time-averaged volume fraction of liquid represents the probability to find liquid in a specific location of the domain during the time of observation. A value of 1 in a volume cell indicates that liquid was present for 100 pct of the time in that cell. $S D$ is therefore identified by filtering out from the distribution the volume below the initial bath heigth and the areas where the time-averaged liquid volume fraction is $<0.005$. The areas with value 0 are excluded since they are not useful for the determination of the liquid splashing area. By doing this, the volume above the free surface with a probability between 0.5 and $100 \mathrm{pct}$ to find liquid during the time of observation is selected. Figures 11(a) and (b) show comparisons of $S D$ at different lance positions and gas flow rates. In agreement with a previous numerical study by the authors ${ }^{[19]}$ and with the experimental observation by Liow et al. ${ }^{[12]}$ the liquid splashing initially increases with the submersion depth before reaching a sort of plateau at deeper lance positions, after which no further changes can be observed. The achievement of an upper bound can be related to several reasons. On one hand, when the injection is deeper in the bath, more energy is lost inside the bath in the bubble-liquid interactions, limiting the disruption when the cavity opens up. From the other, the multiphase flow develops within a finite volume and it is evident that the physical phenomena have lower and upper limits. A similar upper limit should be seen when the gas flow rate is increased. ${ }^{[12,19]}$ However, $S D$ is monotonically enhanced from $Q_{1}$ to $Q_{3}$, probably because of the constrained range of variation in the flow rate. It is interesting to observe that, apart from the lower lance positions, the immersion depth is a more effective control parameter than the gas flow rate when determining a certain splashing level.

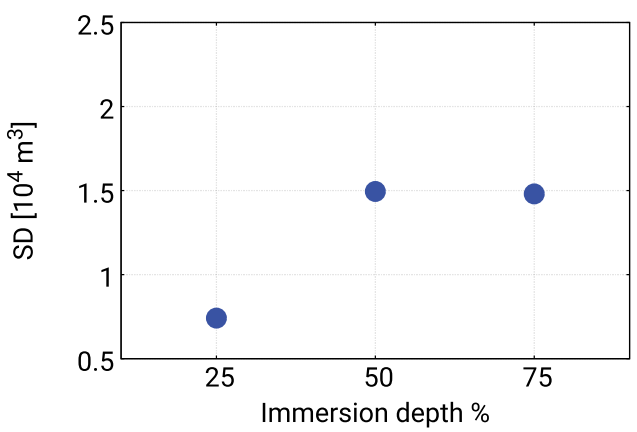

(a)
The gas-liquid interface is identified with an iso-surface at $\alpha_{\text {liq }}=0.5$, and it represents the contact area between the liquid and the gas phases, taking into account the free surface, the bubbles in the liquid phase, and the droplets in the gas phase. The overall interface area is calculated by tracking the integral of this interface area over time and averaging it for the 20 seconds range of observation. Figures 12(a) and (b) show the time-averaged interface area at different lance positions and gas flow rates. An increase is observed when the lance is lowered and more gas is injected. Although the splashing does not change from the middle to the bottom configuration, the interface area is still enhanced, because larger bubbles detach from the lance and stronger gas entrainment takes place. Both control parameters can therefore act on the determination of a certain gas-liquid interface, which is crucial for the kinetics of TSL smelting processes.

\section{CONCLUSIONS}

The present work focused on the dynamics of a TSL metal bath in cylindrical vessels. A previous work by the authors, together with other studies in the literature, suggested the possible presence of rotational waves under certain circumstances. To investigate this phenomenon, a specific post-processing procedure was applied to the simulation results of a validated VOF-based CFD model. The outcomes of the study can be summarized as follows:

- Rotational sloshing due to top-submerged gas injection was detected in the metal bath. Using CFD, it is possible to actually see that the synchronism between the bubbles and free surface motion develops as theorized by Schwarz. ${ }^{[8]}$ It is shown that under this condition of resonance, rotational waves are sustained by the gas injection.

- CFD allows these sloshing phenomena to be directly calculated, for example, for the induced swirl velocity in the liquid bath. The simulation results were compared to an analytical expression proposed by Hutton, showing very good agreement. The sloshing and the induced swirl velocity increase when the lance is

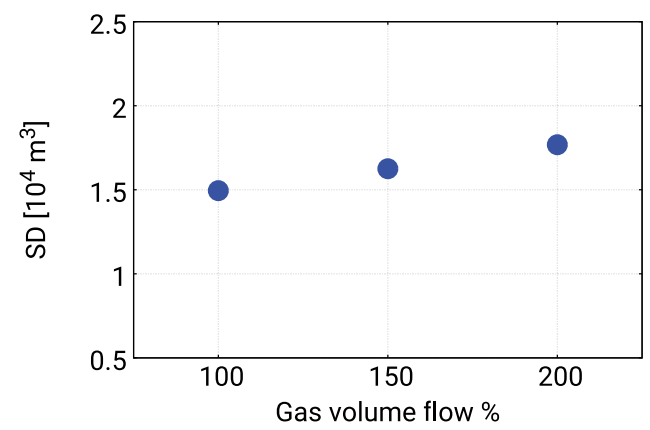

(b)

Fig. 11-Time-averaged splashing domain at different lance immersion depths $(a)$ and gas flow rates $(b)$. 


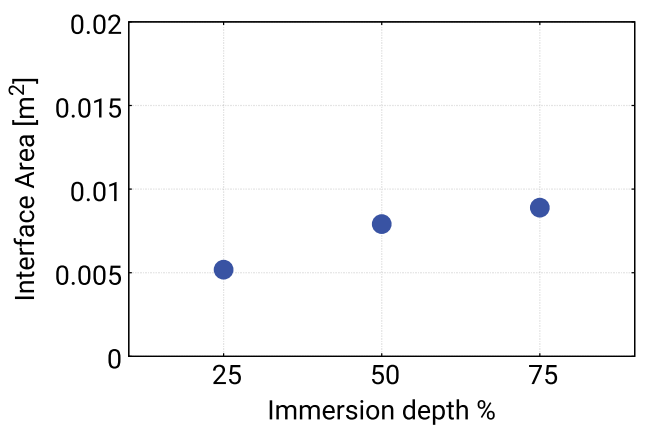

(a)

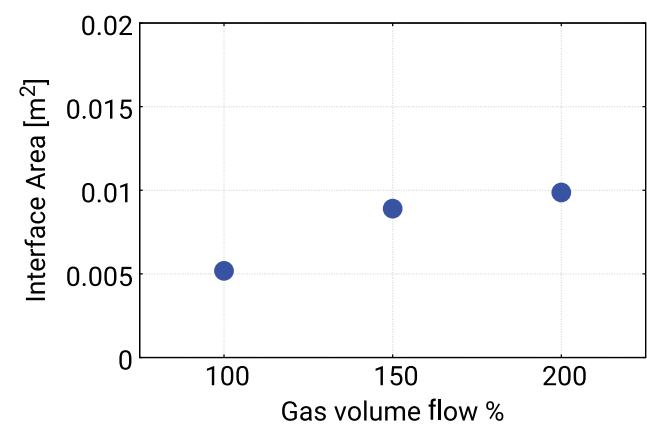

(b)

Fig. 12-Time-averaged gas-liquid interface area at different lance immersion depths $(a)$ and gas flow rates $(b)$.

lowered into the melt and the gas flow rate is increased. An upper limit for the resonance condition appeared at the highest flow rate, as also found by Liow et al. ${ }^{[12]}$ The same was not observed for the lance positioning. However, the author believes that a further step in the submersion of the lance would lead to a sloshing disruption. As previously discussed, it is relevant to point out that understanding these phenomena in detail contributes to understand the overall design of TSL plants, from the lance system to the building foundations.

- Further results concerning the liquid splashing and the overall interface area confirm the importance of the lance submersion and the gas flow rate as control parameters.

- The liquid alloy used in the present work still has a low viscosity compared to that of typical fayalitic slags. To gain a better understanding of the sloshing phenomenon in real TSL smelters, a further step should consider a typical slag bath, where the rotational flows might be damped by the high viscous stresses. Other damping factors might be given by the solid fraction in the bath, coming from both the feed stream and the formation of magnetite, and by the presence of a slag foam layer. ${ }^{[20]}$ This leaves room for future investigations.

\section{ACKNOWLEDGMENTS}

The authors thank the Center for Information Services and High Performance Computing (ZIH) at TU Dresden for the allocation of the computing time. The German Federal Ministry of Education and Research (BMBF) has funded this research within the framework of the Center for Innovation Competence Virtuhcon (Virtuhcon II, project no. 03Z22FN11).

\section{FUNDING}

Open Access funding enabled and organized by Projekt DEAL.

\section{OPEN ACCESS}

This article is licensed under a Creative Commons Attribution 4.0 International License, which permits use, sharing, adaptation, distribution and reproduction in any medium or format, as long as you give appropriate credit to the original author(s) and the source, provide a link to the Creative Commons licence, and indicate if changes were made. The images or other third party material in this article are included in the article's Creative Commons licence, unless indicated otherwise in a credit line to the material. If material is not included in the article's Creative Commons licence and your intended use is not permitted by statutory regulation or exceeds the permitted use, you will need to obtain permission directly from the copyright holder. To view a copy of this licence, visit http://creativec ommons.org/licenses/by/4.0/.

\section{SUPPLEMENTARY INFORMATION}

The online version contains supplementary material available at https://doi.org/10.1007/s11663-021-02182-x.

\section{REFERENCES}

1. Y. Kato, K. Nakanishi, T. Nozaki, K. Suzuki, and T. Emi: Trans. Iron Steel Inst. Jpn., 1985, vol. 25, pp. 459-66.

2. M. Iguchi, T. Uemura, H. Yamaguchi, T. Kuranga, and Z. Morita: ISIJ Int., 1994, vol. 34 (12), pp. 973-79.

3. H. Madarame, K. Okamoto, and M. Iida: J. Fluids Struct., 2002, vol. 16 (3), pp. 417-33.

4. M.S. Lee, SL. O'Rourke, and N.A. Molloy: Scand. J. Metall., 2003, vol. 32 (5), pp. 281-88.

5. Y. Eisaku, S. Yusuke, S. Tatsuo, in: MATEC Web Conf., vol. 211, p. 15003 .

6. L. Shui, Z. Cui, X. Ma, M.A. Rhamdhani, AV. Nguyen, and B. Zhao: Metall. Mater. Trans. B, 2016, vol. 47B (1), pp. 13544.

7. R.E. Hutton: J. Appl. Mech., 1964, pp. 123-30.

8. M.P. Schwarz: Chem. Eng. Sci., 1990, vol. 45 (7), pp. 1765-77.

9. M.P. Schwarz: Appl. Math. Model., 1996, vol. 20 (1), pp. 4151. 
10. P.S. Arthur, S.P. Hunt: in: John Floyd International Symposium on Sustainable Developments in Metals Processing, July.

11. J.L. Liow and N.B. Gray: Metall. Trans. B, 1990, vol. 21 (6), pp. $987-96$.

12. J.L. Liow, W.H.R. Dickinson, M.J. Allan, and N.B. Gray: Metall. Trans. B, 1995, vol. 26 (August), pp. 887-89.

13. J.L. Liow, N.B. Gray: in: 11th Australasian Fluid Mechanics Conference, pp. 643-46.

14. Y. Wang, L. Cao, M. Vanierschot, Z. Cheng, B. Blanpain, and M. Guo: Chem. Eng. Sci., 2020, vol. 212, p. 115359.

15. D. Obiso, M. Akashi, S. Kriebitzsch, B. Meyer, M. Reuter, S. Eckert, and A. Richter: Metall. Mater. Trans. B, 2020, vol. 51B (4), pp. 1509-25.
16. M. Akashi, O. Keplinger, N. Shevchenko, S. Anders, M.A. Reuter, and S. Eckert: Metall. Mater. Trans. B, 2020, vol. 51B, pp. 124-39.

17. E. Mounsey, H. Li, J.W. Floyd: in: Copper 99 - TMS Conference.

18. S. aus der Wiesche: Comput. Mech., 2003, vol. 30, pp. 374-87.

19. D. Obiso, S. Kriebitzsch, M. Reuter, and B. Meyer: Metall. Trans. B, 2019, vol. 50B, pp. 2403-20.

20. A. Sauret, F. Boulogne, J. Cappello, E. Dressaire, and H.A. Stone: Phys. Fluids, 2015, vol. 27, p. 022103.

Publisher's Note Springer Nature remains neutral with regard to jurisdictional claims in published maps and institutional affiliations. 